\title{
COVID-19 and purinergic signaling: the need for investigation
}

\author{
Andréia Machado Cardoso ${ }^{1}$ \\ Received: 16 June 2020 / Accepted: 6 July 2020 / Published online: 13 July 2020 \\ (C) Springer Nature B.V. 2020
}

Keywords Purinergic signaling · COVID-19 · Coronavirus

This letter aims to raise the need for investigation about the role of purinergic signaling in Coronavirus disease 19 (Covid19) pathophysiology. Moreover, experimental model tests with P2X blockers, P1 agonists, and/or enzyme inhibitors could be useful to unveil the possible mechanisms underlying COVID-19 physiopathology related to the role of purinergic system components.

From now, what we know is that acute respiratory issues are the primary cause of mortality of Coronavirus disease 19 (Covid-19) patients. No effective treatment is available for instance, and even the disease pathophysiology is poorly understood. Respiratory distress is caused and sustained by a massive release of proinflammatory cytokines that triggers an uncontrolled inflammation accompanied by lung edema and disseminated coagulation [5].

The regulation of the immune system cells and platelets functions plays a central role in COVID-19 disease [5]. Nowadays it is known that purinergic signaling can modulate platelet activation, cellular trafficking of immune cells as well as the release of pro- and antiinflammatory cytokines [2, 4]. Recently, it has been proposed that P2X7 antagonist administration could be an option for critically ill Covid-19 patients due its special role in the release of cytokines by macrophages [1]. It has also been suggested that therapeutic benefit of purinergic modulators, such as dipyridamole (A2A receptor agonist), must be investigated because it targets multiple pathways that have been implicated in COVID-19 pathogenesis [3]. Indeed, Kanthi research group is developing protocols to launch multiple clinical trials with dipyridamole at different stages of COVID-19 (NCT04391179, available on http:// clinicaltrials.gov) [3]. These two recent published papers

Andréia Machado Cardoso

andreia.cardoso@uffs.edu.br; deiaa.mc@gmail.com

1 Federal University of Fronteira Sul, Medicine Course, Graduate Program in Biomedical Sciences, Member of the Brazilian Purine Club, Campus Chapecó, Chapecó, SC, Brazil highlight the importance of investigating purinergic system in COVID-19 physiopathology.

It is important to note that blood extracellular purines and adenosine, components of the purinergic system, are increasingly recognized as key mediators of activation and/or inhibition of circulating immune cells and platelets adhesion. Since ATP and adenosine, as well as other nucleotides, play a role in immune system and platelet activation and have opposite effects, proper regulation of purinergic signaling by ectonucleotidases and adenosine deaminase (ADA) may be crucial to modulate immune cells and platelet functions $[2,4]$. Once released by a damaged tissue into the blood, ATP can be broken down by a cascade of ectonucleotidases into ADP, AMP, and adenosine. By regulating the concentrations of extracellular nucleotides and nucleosides, especially ATP and adenosine, different combinations of ectonucleotidases would therefore be expected to have distinct effects on immune cells and platelet performance. Indeed, there are several ectonucleotidases, and each of them differs in its biochemical properties, including substrate affinity/preference, formation of metabolites, and optimal $\mathrm{pH}$ of activity. All immune cells and platelets express ectonucleotidases and ADA at different levels.

Signals delivered by extracellular ATP and adenosine are detected and transduced by $\mathrm{P} 2$ and $\mathrm{P} 1$ receptors, respectively. Virtually all immune cells and platelets express P2 and P1 receptors; thus, purinergic signaling affects all aspects of immunity and inflammation as well as of platelet aggregation [1-5]. From what we know about purinergic cascade and inflammation, it is possible to infer that in COVID-19 patients, we have upregulation of $\mathrm{P} 2 \mathrm{X} 7$, for example, that is helping macrophages and lymphocytes to produce and release a large amount of proinflammatory cytokines. Thus, this letter encourages the researchers to analyze different aspects of purinergic signaling in both human and experimental model. For example, to analyze lung infiltrated of immune cells as well as circulating immune cells in COVID-19 patients could be very useful to better understand the involvement of purinergic signaling in this disease. 


\section{Compliance with ethical standards}

Conflicts of interest Andréia Machado Cardoso declares that she has no conflict of interest.

Ethical approval This article does not contain any studies with human participants or animals performed by any of the authors.

\section{References}

1. Di Virgilio F, Tang Y, Sarti AC, Rossato M. A rationale for targeting the P2X7 receptor in coronavirus disease 19 (Covid-19) Br J Pharmacol. 2020; https://doi.org/10.1111/bph.15138. Online ahead of print
2. Di Virgilio F, Vuerich M (2015) Purinergic signaling in the immune system. Auton Neurosci 191:117-123

3. Kanthi Y, Knight JS , Zuo Y, Pinsky DJ. New (re)purpose for an old drug: purinergic receptor blockade may extinguish the COVID-19 thrombo-inflammatory firestorm. 2020 Jun 12;140971. https://doi. org/10.1172/jci.insight.140971. Online ahead of print

4. Morrell CN, Aggrey AA, Chapman LM, Modjeski KL (2014) Emerging roles for platelets as immune and inflammatory cells. Blood 123:2759-2767

5. Tamimi F, Abusamak M, Akkanti B, Chen Z, Yoo SH, KarmoutyQuintana $\mathrm{H}$. The case for chronotherapy in COVID-19 induced acute respiratory distress syndrome (ARDS). Br J Pharmacol. 2020; https://doi.org/10.1111/bph.15140. Online ahead of print

Publisher's note Springer Nature remains neutral with regard to jurisdictional claims in published maps and institutional affiliations. 